\title{
Nosocomial Staphylococcal Toxic Shock. Case Report
}

\author{
Manuela Arbune ${ }^{1,2 *}$, Loredana Tercu² \\ 1 "Dunarea de Jos" University Galati, Romania \\ 2 Hospital of Infectious Diseases "Sf. Cuvioasa Parascheva" Galaţi, Romania
}

\begin{abstract}
Staphylococcal toxic shock syndrome (STSS) is a rare, potentially lethal infection, with a clinical picture of multiple organ dysfunction and shock. The etiology is Staphylococcus aureus exotoxin, while enterotoxins act as superantigens. Most cases are identified in women using a vaginal tampon. A 51-year-old female, with a past medical history of biliary dyskinesia, presented in the emergency room complaining of sudden onset of abdominal pain, vomiting, headache, myalgia, and chills. The initial diagnosis was biliary colic and was treated parenterally with Amoxi-clavulanate and fluid replacement. Initially, progress was unsatisfactory. Four days after admission she developed a systemic inflammatory syndrome, diffuse rash, jaundice, oliguria, confusion, persistent hypotension and biological evidence of thrombocytopenia, nitrogen retention, and cholestasis. She was admitted to the intensive care unit. A gluteal phlegmon induced after intramuscular injections was identified and surgically treated. Blood bacteriological cultures were negative, though MRSA was isolated in phlegmon pus. A diagnosis of STSS was based on CDC criteria.
\end{abstract}

The risks of similar infections could be prevented by limiting intramuscular treatments and monitoring invasive procedures.

Keywords: staphylococcal toxic shock syndrome, MRSA, phlegmon

Received: 04 April 2016 / Accepted: 26 May 2016

\section{INTRODUCTION}

The first description of staphylococcal toxic shock syndrome (STSS) in children dates back to 1978 [1]. However, most early reports, related STSS to menstruating women and the use of absorbent tampons with synthetic fibers [2]. There are, however, many other causes of infection, including nosocomial environmental conditions, and STSS continues to represent a public health problem [3].

The criteria for defining STSS are high fever, rash, hypotension, multi-organ failure involving at least three or more systems, and desquamation following the onset of acute illness [4].

While STSS is potential lethal, the survival rate depends on early recognition, source control, and antibiotic administration. Both clindamycin and intravenous immunoglobulin therapy improved the prognostic of STSS, according to recent studies [5].

\section{CASE REPORT}

A 51-year-old menopausal woman, with a past medical history of biliary dyskinesia, presented in the emergency room after a party. She complained of the sudden onset of abdominal pain, bilious vomiting, headache and chills. A diagnosis of calculous biliary colic was based on physical and ultrasound examination. She received intramuscular analgesic, antispasmodic medication, intravenous Amoxi-clavulanate and fluid replacement. On the fourth day after admission, she developed jaundice, oliguria, and confusion. Biological data evidenced thrombocytopenia, nitrogen retention, increasing cytolysis, and cholestasis.

Infectious diseases opinion was sought to eliminate a diagnosis of acute viral hepatitis or leptospirosis (Table 1). On clinical examination, she was seen to be disorientated, pyretic $\left(39.3^{\circ} \mathrm{C}\right)$, with tachycardia (heart rate, $140 / \mathrm{min}$ ), polypnea (respiratory rate $32 / \mathrm{min}$ ), jaundice and a diffuse rash, although her extremities were cold

\footnotetext{
Correspondence to: Manuela Arbune, Hospital of Infectious Diseases "Sf. Cuvioasa Parascheva" Galați, Romania, Traian street no. 393, Phone: +40236 $334022,+40236$ 334032. 
and cyanotic. Low systolic blood pressure $(80 \mathrm{~mm} \mathrm{Hg})$ was persistent despite fluid resuscitation and she required vasopressor support. Empirical antibiotic treatment with vancomycin and meropenem, corticosteroids and intravenous immunoglobulin treatment were justified by the evidence of septic shock of unknown origin with multi-organ dysfunction, associated with a high probability of a nosocomial etiology.

A gluteal phlegmon, occurring after intramuscular injections, was identified on clinical re-examination and was surgically debrided and drained. Diagnosis of TSS was based on CDC criteria (Table 2). Blood bacteriological cultures were negative, but MRSA was isolated from the phlegmon pus, which was susceptible to vancomycin and linezolid. Desquamation from palms was recorded after a week, but the patient' condition resolved and she was discharged after 18 days.

\section{DISCUSSION}

Although effective anti-staphylococcal antibiotics are available, Staphylococcus aureus is one of the main causes of morbidity and mortality worldwide. An estimated $20 \%$ of the general populations are Staphylococcus aureus carriers, though skin colonization is usually brief and repetitive. The pathophysiology of STSS is related to Staphylococcus aureus exotoxins, mainly enterotoxin Band TSS toxin type-1 (TSST-1). Toxins function as highly active superantigens, trigger immune cell activation and release cytokines that are involved in the severe inflammatory response. Contrary to the normal immune pathway, superantigens are not processed by antigen presenting cells and widespread activation of T-lymphocytes. Consequently, massive cytokine are released, leading to septic shock and tissue destruction [6].

The risk factors for STSS are colonization with toxigen Staphylococcus aureus and lack of antitoxic immunity. Differential diagnosis of TSS includes Streptococcal Toxic Shock Syndrome, severe sepsis with Gram Negative germs, leptospirosis, rickettsial diseases, viral exanthema, vasculitis, and drug reactions [7].

Recently cases of STSS have been reported in men, women, adults and children. They were associated with various circumstances: surgical procedures such as nasal packing, use of catheters or other devices, nonsurgical focal infections such as cellulitis, subcutaneous abscesses, infected burns, pneumonia, infections of joint and bones, and non-medical procedures, as tattoos and intravenous drugs use [8].

Table 1. Female, $\mathbf{5 1}$ years old: Laboratory data related to TSS

\begin{tabular}{|c|c|}
\hline Lab - Investigations & Patient Lab Data \\
\hline WBC $(\times 103 / \mu \mathrm{L})$ & 15.13 (NV: 4-10) \\
\hline Neutrophiles (\%) & 11.76 (NV: 2-8) \\
\hline Platelets $(x 103 / \mu \mathrm{L})$ & 49 (NV: 150-450) \\
\hline $\mathrm{ESR}(\mathrm{mm} / \mathrm{h})$ & 50 (NV: 6-13) \\
\hline Glicemia (mg/dl) & 58 (NV: 70-100) \\
\hline Urea $(\mathrm{mg} / \mathrm{dl})$ & $195.85(\mathrm{NV}<40)$ \\
\hline Total Bilirubin (mg/dl) & 12.96 (NV: 0.2-9.8) \\
\hline Alk phosphatase (U/I) & 287.92 (NV: 20-115) \\
\hline $\operatorname{ALT}(\mathrm{U} / \mathrm{I})$ & 45.7 (NV: 0-39) \\
\hline AST $(U / I)$ & 46,1 (NV: 0-42) \\
\hline CPK (U/I) & $742(\mathrm{NV}<192)$ \\
\hline HBsAg & negative \\
\hline $\mathrm{HBc}-\lg \mathrm{M}$ & negative \\
\hline HVA-IgM & negative \\
\hline $\mathrm{HVC}-\mathrm{Ab}$ & negative \\
\hline HIV-Ab (ELISA) & negative \\
\hline Lepstospira-IgM (ELISA) & negative \\
\hline Blood cultures & negative \\
\hline Nasal swab culture & negativeStaphylococcusspp \\
\hline
\end{tabular}

Table 2: Female, $\mathbf{5 1}$ years old: Clinical criteria of TSS CDC Criteria* Patient Clinic Data

1. Fever

2. Rash

3. Desquamation

4. Hypotension

5. Multisystem involvement

\begin{tabular}{lc}
\hline gastrointestinal & vomiting at onset \\
\hline renal & Urea $\times 4.9$ UVN \\
\hline muscular & $\mathrm{CPK} \times 3.8 \mathrm{UNV}$ \\
\hline hematologic: $\mathrm{Plt}<100000$ & $\mathrm{Plt} 49000 / \mathrm{mm}^{3}$ \\
\hline central nervous system & disorientation \\
\hline
\end{tabular}

*Source: Center for Disease Control and Prevention. 2011 case definition, toxic shock syndrome. Available at http://wwwn.cdc.gov/nndss/conditions/toxic-shock-syndrome-other-than-streptococcal.

The present case is one of nosocomial STSS with a MRSA strain, following intramuscular injections, on the fourth day following admission to hospital.

\section{- CONCLUSIONS}

Intramuscular injections are risk factors for nosocomial STSS. Physical examination of hospitalized patients undergoing parenteral therapy for the prevention of 
nosocomial STSS, should be repeated, including examination of the gluteal region.

Prompt diagnosis, immediate intensive care support, and surgical treatment are crucial for the resolution of STSS.

\section{DONFLICT OF INTEREST}

None declared

\section{REFERENCES}

1. Todd J, Fishaut M, Kapral F. Toxic-shock syndrome associated with phage-group-I Staphylococci. Lancet. 1978;2(8100):11168.

2. David M, Daum R. Community-Associated Methicillin Resistant Staphylococcus aureus: Epidemiology and Clinical Consequences of an Emerging Epidemic. Clin Microbiol Rev. 2010;23:616-87.
3. DeVries AS, Lesher L, Schlievert PM, et al. Staphylococcal toxic shock syndrome 2000-2006: epidemiology, clinical features, and molecular characteristics. PLoS One. 2011;6:e22997.

4. Stevens DL, Bisno AL, Chambers HF, et al. Practice guidelines for the diagnosis and management of skin and soft tissue infections: 2014 update by the infectious diseases society of America. Clin Infect Dis. 2014;59:147-59.

5. Linnér A, Darenberg J, Sjölin J, Henriques-Normark B, NorrbyTeglund A. Clinical Efficacy of Polyspecific Intravenous Immunoglobulin Therapy in Patients With Streptococcal Toxic Shock Syndrome: A Comparative Observational Study. Clin Infect Dis. 2014;59:851-7.

6. Brosnahan AJ, Schlievert PM. Gram-positive bacterial superantigen outside-in signaling causes toxic shock syndrome. FEBS J. 2011;278:4649-67.

7. Herzer CM. Toxic shock syndrome: Broadening the differential diagnosis. J Am Board Fam Pract. 2001;14:131-6.

8. Marton M. Staphylococcal Toxic Shock Syndrome Caused By An Intravaginal Product. A Case Report. J Crit Care Med. 2016;2:51-5. 